\title{
Effect of Levetiracetam Monotherapy on Hematological Parameters in Children with Idiopathic Epilepsy
}

\author{
SARA M. IBRAHIM, M.Sc.*; SAHAR A. ABD EL-AZIZ, M.D.*; KHALED T. MUHAMMAD, M.D.* and \\ HESHAM A. EL-SEROGY, M.D.** \\ The Departments of Pediatric* and Clinical Pathology**, Faculty of Medicine, Tanta University, Egypt
}

\begin{abstract}
Background: Levetiracetam is broad spectrum, second generation antiepileptic drug which is highly tolerated in children with minor adverse events. Studies evaluating the effect of levetiracetam on hematological parameters have reported an unexplained increased incidence of infections such as pharyngitis and rhinitis. There have been case reports of pancytopenia, thrombocytopenia, eosinophilia and lymphopenia. However, cases of isolated low red blood cell counts, hemoglobin and hematocrit as compared to placebo are reported.
\end{abstract}

Aim of Study: This study aimed to evaluate the changes in complete blood picture, prothrombin time, partial thromboplastin time and platelet functions in children with idiopathic epilepsy who were treated with levetiracetam as monotherapy.

Patients and Methods: This study was conducted on fifty children of both sexes presented with idiopathic epilepsy during the period from June 2016 to May 2017. Patients were assessed by monitoring complete blood picture, prothrombin time, partial thromboplastin time and platelet functions before and 6 months after receiving levetiracetam monotherapy.

Results: There were no significant alterations in the evaluated parameters during the study after levetiracetam administration when compared to results before treatment

Conclusion: Levetiracetam monotherapy has proven to be well tolerated in treatment of children with idiopathic epilepsy and does not appear to cause significant changes in complete blood picture, prothrombin time, partial thromboplastin time and platelet functions.

Key Words: Idiopathic epilepsy - Levetiracetam monotherapy - Hematology.

\section{Introduction}

EPILEPSY is a disorder of the brain characterized by an enduring predisposition to generate seizures and by the neurobiologic, cognitive, psychologic, and social consequences of this condition [1]

Correspondence to: Dr. Sara M. Ibrahim, E-Mail: drsaram.ibrahim@gmail.com
Epilepsy is the most common serious neurologic disorder in childhood that often requires lifetime treatment [2]. The aim of epilepsy management is complete seizures cessation and maintenance of a good quality of life, which is affected not only by the epileptic attacks but also by the adverse effect of the drug [3]. Levetiracetam is a major breakthrough in the treatment of epilepsies. It is one of broad spectrum, second generation antiepileptic drugs which is mainly effective in partial onset seizures with or without secondary generalization, myoclonic seizures and primary generalized tonicclonic seizures. Levetiracetam is highly tolerated in children with minor adverse events [4]. Studies evaluating the effect of levetiracetam on hematological parameters have reported an unexplained increased incidence of infections such as pharyngitis and rhinitis [5]. There have been isolated case reports of levetiracetam causing pancytopenia, thrombocytopenia, eosinophilia, lymphopenia [6,7] However, cases of isolated low red blood cell counts, hemoglobin and hematocrit as compared to placebo are reported [8]

\section{Patients and Methods}

The study was carried out at Pediatric Department of Tanta University Hospital in duration of 12 months from June 2016 to the end of May 2017. The study population consisted of 50 children, 23 male and 27 female, with idiopathic epilepsy.

Patients aged from 2 to 15 years old, suffering from idiopathic epilepsy with positive family history and normal brain magnetic resonance imaging study were included. But, patients with symptomatic epilepsy, underlying hematological disorder or patients who received medications known to affect blood parameters were excluded. 
The Research Ethical Committee, Faculty of Medicine, Tanta University approved the design of the study. An informed written consent was taken from each parent or guardians after explanation of benefits and risks. All patients' data was confidential with secret code numbers and was used for the current study only. The study did not harm patients or delay their improvement.

All patients were subjected to: Complete history taking, thorough physical examination, magnetic resonance imaging (MRI) and electroencephalogram (EEG) study. The records of all patients were reviewed and data were collected prospectively.

About $5.5 \mathrm{ml}$ of venous blood were obtained from each patient by venipuncture under complete aseptic condition. Each sample was collected in a specific labeled container with patient's name, serial number and date of taking the sample. About $2 \mathrm{ml}$ blood was put into ethylene diamine tetraacetic acid (EDTA) vacutainer tube for complete blood picture and platelet function assay. Another $1.8 \mathrm{ml}$ blood was put into vacutainer tube containing trisodium citrate $3.2 \%$ (9:1 ratio) for prothrombin and thromboplastin time. Also, about $1.6 \mathrm{ml}$ of blood was put into vacutainer tube containing buffered sodium citrate (4:1 ratio) for platelet functions. These investigations were done for each patient before and 6 months to one year after the start of treatment.

\section{Statistical analysis:}

The collected data were organized, tabulated and statistically analyzed using Statistical Package for Social Studies (SPSS) version 19, released 2010 and created by IBM, Armonk, New York, USA. For numerical values the range, mean and standard deviations were calculated. The differences between two mean values were used using student's dependent $t$-test. For categorical variable the number and percentage were calculated. The level of significant was adopted at $p<0.05$ [9].

\section{Results}

Records and data of all patients were collected prospectively. The data of the patients were statistically analyzed and the results are summarized and tabulated in the following Tables and Figures.

The age of studied patients ranged from 2 to 15 years old with mean value $8.52 \pm 3.82$ years (Table 1). The male patients were 23 and represented $46.0 \%$. Female patients were 27 and represented $54.0 \%$ of cases. Duration of levetiracetam admin- istration to the studied patients ranged from 6 to 12 months with mean value $7.88 \pm 1.81$ months. (Table 2).

This study showed that there were a slight decrease in the mean value of red blood cells (RBCs) count, blood hemoglobin level (HGB) and hematocrit value (HCT) but with no statistically significant differences after levetiracetam administration when compared to results before treatment (Table 3).

As regard to white blood cells (WBCs) count, absolute neutrophil count and platelets count, there were also slight decreases in the mean values but with no statistically significant differences after levetiracetam administration when compared to results before treatment (Table 3 ).

However, there were no statistically significant changes in mean corpuscular volume (MCV), mean corpuscular hemoglobin $(\mathrm{MCH})$ and mean corpuscular hemoglobin concentration (MCHC) after levetiracetam administration when compared to results before treatment (Table 3 ).

Also absolute lymphocyte count, absolute eosinophil count, absolute basophil count and absolute monocyte count showed no statistically significantdifference after levetiracetam treatment when compared to results before treatment (Table 3).

Regarding to prothrombin time (PT), partial thromboplastin time (PTT), adenosine diphosphate, ristocetin and collagen induced platelet functions there were no statistically significant changes after levetiracetam administration when compared to results before treatment (Table 3 ).

Table (1): Distribution of studied patients by age in years.

\begin{tabular}{lcc}
\hline Age (years) & Number & $\%$ \\
\hline$<5$ & 12 & 24.0 \\
$5-<10$ & 18 & 36.0 \\
$10-15$ & 20 & 40.0 \\
Range & \multicolumn{3}{c}{$2-15$} \\
Mean \pm SD & \multicolumn{3}{c}{$8.52 \pm 3.82$} \\
\hline
\end{tabular}

Table (2): Distribution of studied patients by duration of levetiracetam administration in months.

\begin{tabular}{lcc}
\hline Duration (months) & Number & $\%$ \\
\hline $6-<8$ & 23 & 46.0 \\
$8-<10$ & 17 & 34.0 \\
$10-12$ & 10 & 20.0 \\
Range & \multicolumn{3}{c}{$6-12$} \\
Mean \pm SD & \multicolumn{3}{c}{$7.88 \pm 1.81$} \\
\hline
\end{tabular}


Table (3): Changes in hematological parameters in 50 children with epilepsy during levetiracetam monotherapy.

\begin{tabular}{|c|c|c|c|}
\hline Measured parameters & $\begin{array}{l}\text { Mean value before } \\
\text { levetiracetam }\end{array}$ & $\begin{array}{c}\text { Mean value after } \\
\text { levetiracetam }\end{array}$ & $\begin{array}{c}p- \\
\text { value }\end{array}$ \\
\hline Red blood cells (x10 $\left.{ }^{6}\right)($ cell $/ \mathrm{cmm})$ & $4.80 \pm 0.3$ & $4.71 \pm 0.51$ & 0.058 \\
\hline Hemoglobin (gm/dl) & $11.81 \pm 0.38$ & $11.70 \pm 0.62$ & 0.054 \\
\hline Hematocrit value (\%) & $35.98 \pm 1.02$ & $35.73 \pm 1.18$ & 0.059 \\
\hline Mean corpuscular volume (fl) & $80.92 \pm 2.83$ & $85.10 \pm 3.68$ & 0.702 \\
\hline Mean corpuscular hemoglobin (pg) & $29.73 \pm 1.24$ & $29.71 \pm 1.41$ & 0.926 \\
\hline Mean corpuscular hemoglobin concentration (\%) & $33.99 \pm 0.51$ & $33.97 \pm 0.87$ & 0.858 \\
\hline White blood cells $\left(\times 10^{3}\right)($ cell $/ \mathrm{cmm})$ & $6.27 \pm 1.16$ & $5.84 \pm 1.95$ & 0.056 \\
\hline Absolute lymphocyte count (cell/cmm) & $3158 \pm 788$ & $3149 \pm 847$ & 0.908 \\
\hline Absolute neutrophil count (cell/cmm) & $2790 \pm 621$ & $2604 \pm 963$ & 0.090 \\
\hline Absolute eosinophil count (cell/cmm) & $86.92 \pm 61.91$ & $86.56 \pm 77.05$ & 0.980 \\
\hline Absolute basophil count (cell/cmm) & $22.12 \pm 21.27$ & $22.18 \pm 21.06$ & 0.985 \\
\hline Absolute monocyte count (cell/cmm) & $216.38 \pm 138.09$ & $219.82 \pm 137.6$ & 0.814 \\
\hline Platelet count $\left(\mathrm{x} 10^{3}\right)(\mathrm{cell} / \mathrm{cmm})$ & $231.07 \pm 59.94$ & $226.12 \pm 65.86$ & 0.076 \\
\hline Prothrombin time (sec) & $12.3 \pm 0.87$ & $12.56 \pm 1.59$ & 0.058 \\
\hline Partial thromboplastin time (sec) & $30.99 \pm 5.34$ & $32.75 \pm 8.82$ & 0.061 \\
\hline Collagen-induced function (\%) & $73.94 \pm 7.17$ & $71.12 \pm 11.64$ & 0.124 \\
\hline Ristocetin-induced functions (\%) & $75.56 \pm 7.40$ & $73.46 \pm 7.99$ & 0.163 \\
\hline Adenosine diphosphate-induced functions (\%) & $75.02 \pm 7.35$ & $72.58 \pm 13.40$ & 0.278 \\
\hline
\end{tabular}

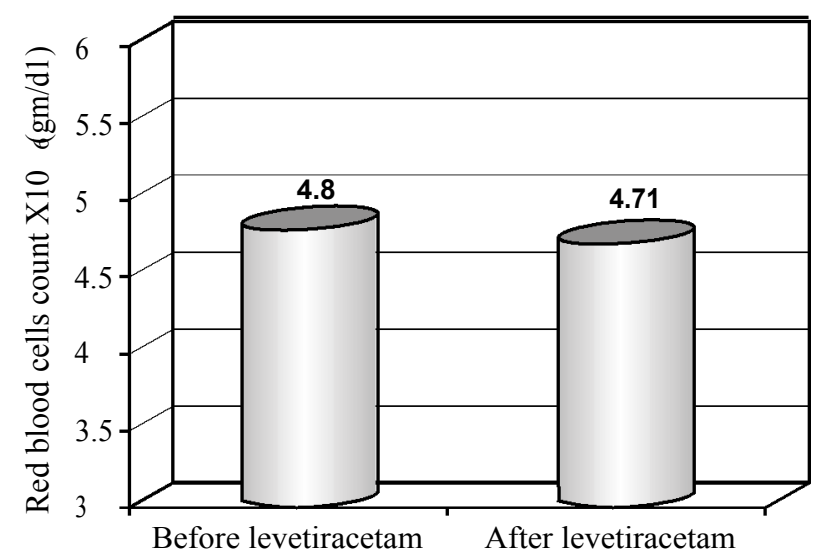

Fig. (1): Comparison between the mean value of red blood corpuscle (RBCs) before and after levetiracetam administration.

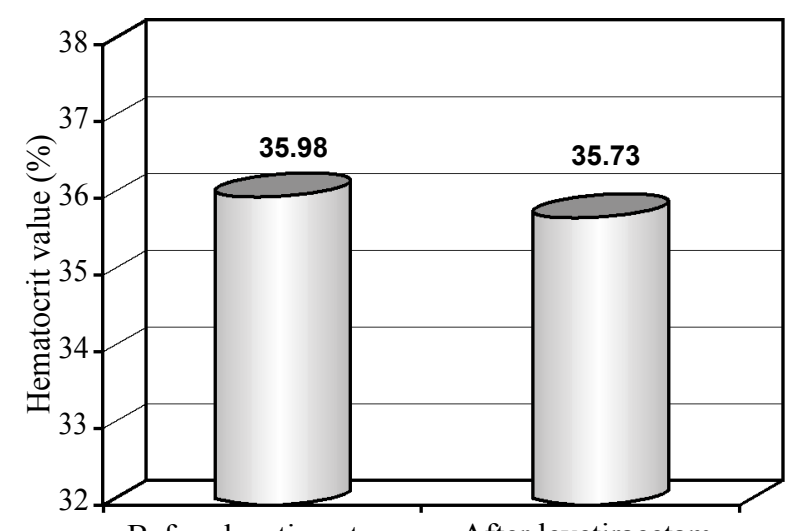

Before levetiracetam After levetiracetam

Fig. (3): Comparison between the mean value of hematocrit value before and after levetiracetam administration.

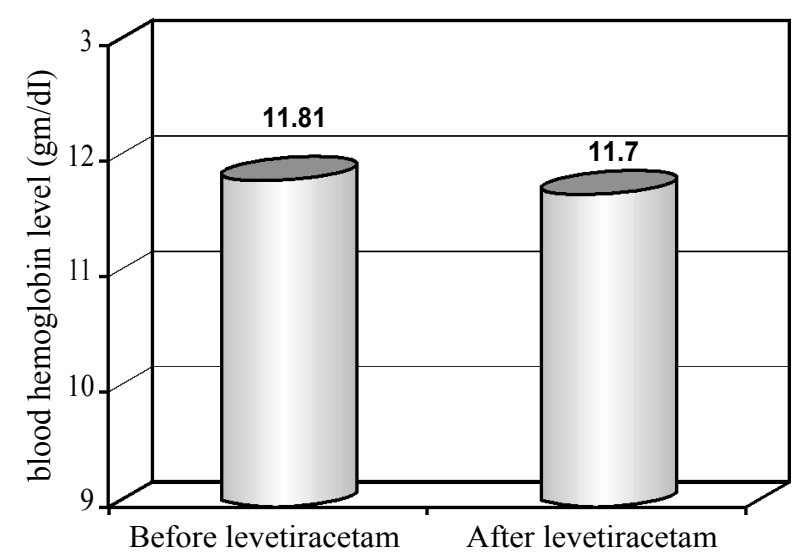

Fig. (2): Comparison between the mean value of blood hemoglobin before and after levetiracetam administration.

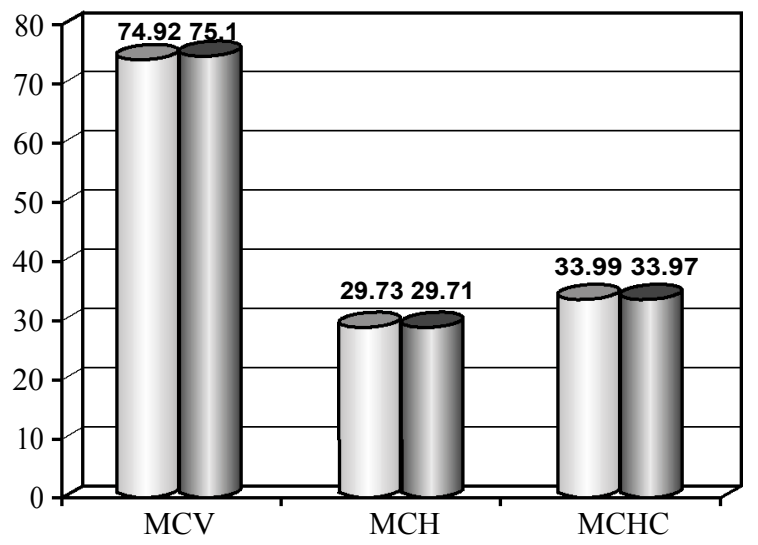

Fig. (4): Comparison between the mean value of red blood cells indices before and after levetiracetam administration. 


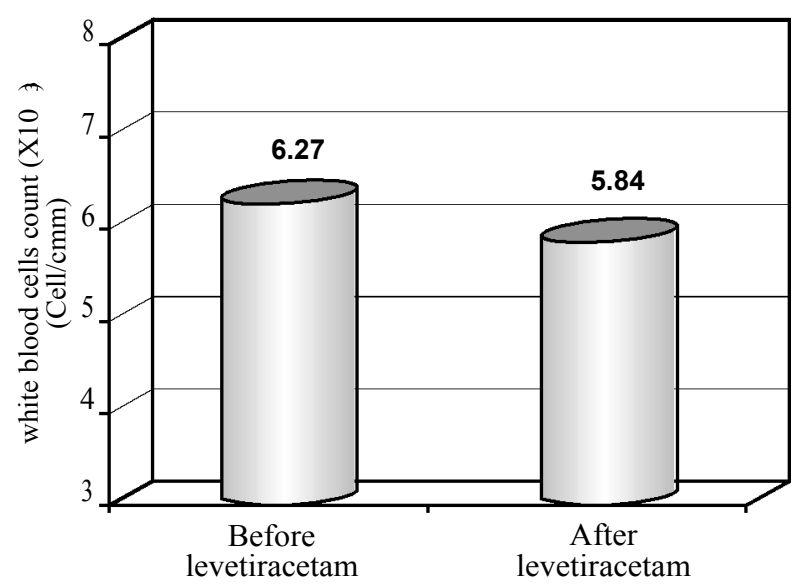

Fig. (5): Comparison between the mean value of white blood cells (WBCs) before and after levetiracetam administration.

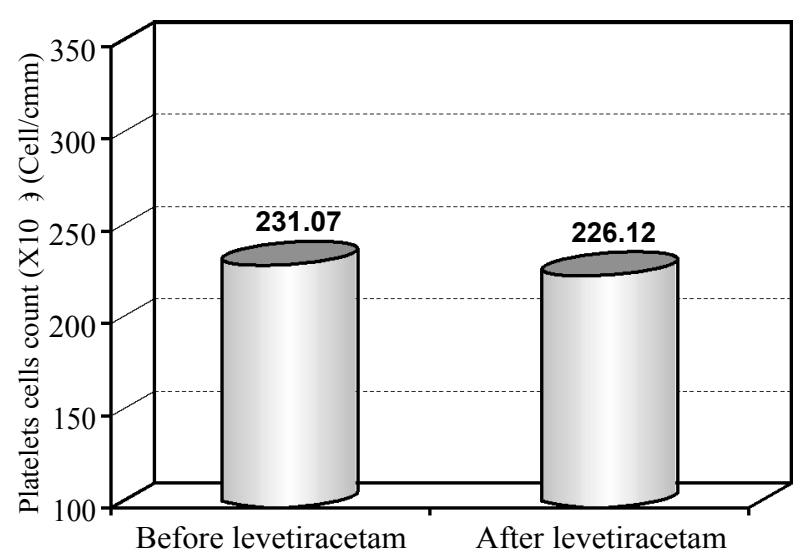

Fig. (7): Comparison between the mean value of blood platelets count before and after levetiracetam administration.

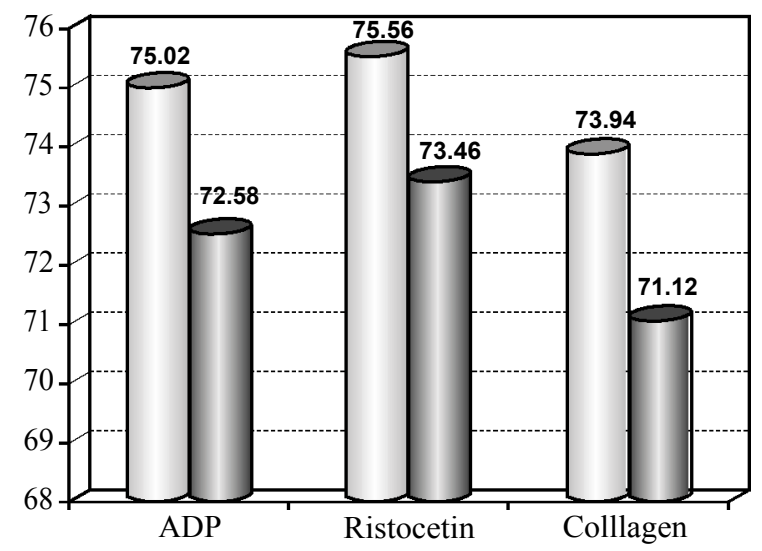

Fig. (9): Comparison between the mean value of platelet functions before and after levetiracetam administration.

\section{Discussion}

Our study showed that there were no statistically significant changes regarding the mean value of red blood cells count, hemoglobin, hematocrit value, red blood cells indices, white blood cells count, differential leucocyte count and platelet

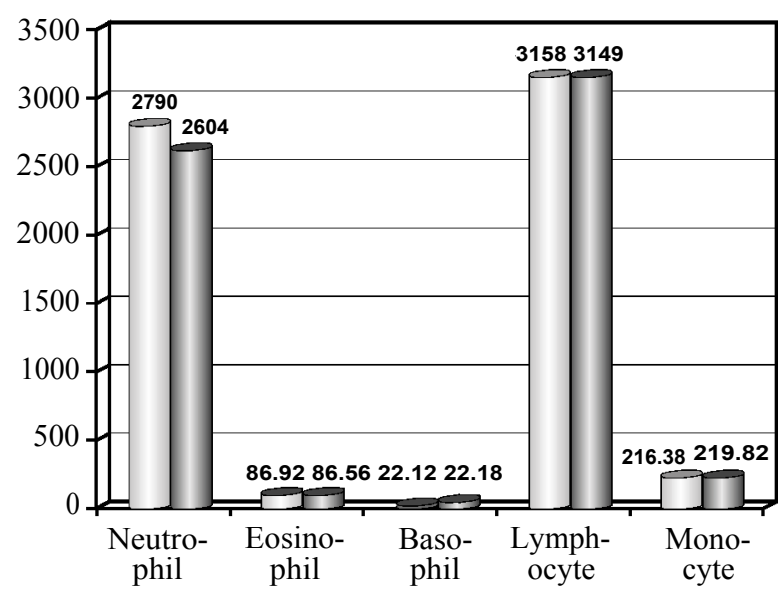

Fig. (6): Comparison between the mean value of differential leucocyte count before and after levetiracetam administration.

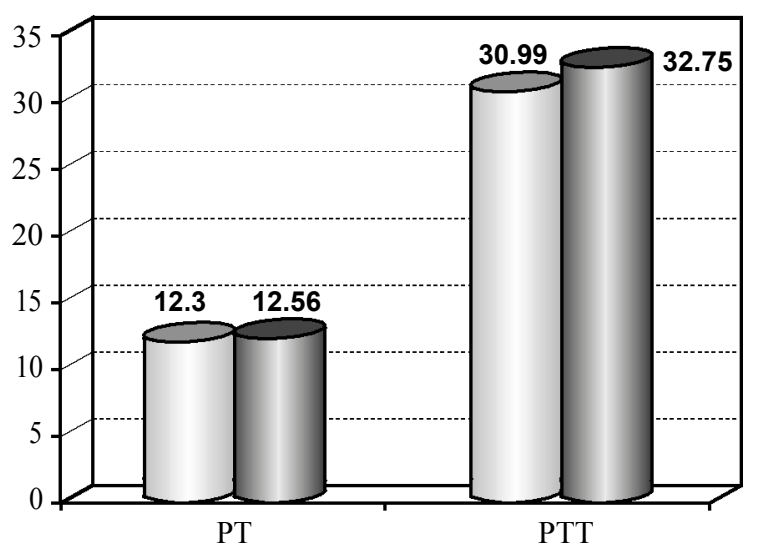

Fig. (8): Comparison between the mean value of prothrombin time and partial thromboplastin time before and after treatment with levetiracetam.

count after levetiracetam administration when compared to results before treatment.

These results were in line with the study done by Dinopoulos et al., [10] that was conducted prospectively on 22 children with idiopathic epilepsy, from 2-15 years old. The study aimed to evaluate the effect of levetiracetam monotherapy on hematological parameters. The evaluation was done before, 2 months and 6 months after levetiracetam intake. They concluded that there were no significant alterations in complete blood picture parameters between baseline and final follow-up except for lymphocyte count.

Dinopoulos et al., [10] stated that there were statistically significant decreases for the mean value of lymphocyte count after 6 months of levetiracetam administration from baseline. The obtained $p$-value was 0.019 .

But, this result was conflicted by study done by Tacke et al., [11] Their trial was a randomized 
controlled trial which compared the effect of levetiracetam and another antiepileptic drug (sulthiame) on lymphocyte count. The study was conducted on 44 children with benign epilepsy with centrotemporal spikes in childhood, 21 of them were levetiracetam-treated and 23 were sulthiametreated. Tacke et al., [11] concluded that there were no significant alterations in the mean value of lymphocyte count in both groups with $p$-value $=$ 0.034 .

Our results were also in agreement with the study done by Kanemura et al., [12], who conducted their study on 61 patients, aged from 16 months to 18 years old, to evaluate the efficacy and safety of levetiracetam in patients with refractory epilepsy. The evaluation was done before and 6 months after levetiracetam administration. The included subjects suffered from any type of epilepsy. Symptomatic or idiopathic epilepsies are included. Also, generalized, focal or unclassified epilepsies are included and the patients were receiving concomitant antiepileptic therapy. Their study concluded that there were no significant alterations in complete blood picture parameters between baseline and final follow-up.

This is also in accordance to Glauser et al., [13] study, which was a randomized, placebo-controlled and double blinded study. It was conducted on 198 patients, 101 on levetiracetam and 97 on placebo, to evaluate efficacy and tolerability of levetiracetam as adjunctive therapy in focal seizures. The age of patients ranged from 4-16 years old. They concluded that there were no significant alterations in complete blood picture parameters except for lymphocyte count between the two studied groups.

Glauser et al., [12] study stated that there were statistically significant changes for the mean value of lymphocyte count after 6 months of levetiracetam administration from baseline with $p$-value $=0.0003$. These results may be assigned to the large sample size (198 patients), the presence of comparison group and the polytherapy administration.

As regard to prothrombin time (PT) and partial thromboplastin time (PTT), our study showed that there were no statistically significant changes after levetiracetam administration when compared to results before treatment.

Our results were in line with Rüegg et al., [14] study, which was retrospective study that reviewed the medical charts of 50 critically ill patients treated with intravenous levetiracetam to evaluate the safety and efficacy of intravenous levetiracetam. The studied patients aged $>18$ years old, $32 \%$ of them were females and $68 \%$ were males. Intravenous levetiracetam was given as an add-on therapy. The aim of levetiracetam administration was treatment from status epilepticus in 24 patients (48\%), treatment of focal or generalized seizures in 19 patients (38\%), and seizure prophylaxis in 7 patients (14\%). The idiopathic seizures represented $2 \%$ and the remaining was symptomatic seizures. Duration of intravenous levetiracetam treatment was from 1-5 days. Rüegg et al., [14] stated in their study that there were no significant alterations in prothrombin time (PT) or partial thromboplastin time (PTT) after levetiracetam treatment when compared to value before treatment.

On the other hand, the study was done by Manohar et al., [15] highlighted that prothrombin time (PT) and partial thromboplastin time (PTT) was prolonged slightly and significantly postoperative, with $p$-value $=0.001$ for both, when compared to preoperative results, although the values remained within normal limits. Manohar et al., [15] was a retrospective study, conducted on 84 patients aged from 11-15 years old. The study aimed to evaluate the effect of levetiracetam adjunctive therapy on coagulation and bleeding in perioperative period of epilepsy surgery. These results may be attributed to multiple combinations of antiepileptic drugs in studied patients and expression of data as median.

As regard to platelet functions, our study showed that there were no statistically significant changes after levetiracetam administration when compared to results before treatment.

The pediatric case report presented by Herrman et al., [16] was in line with these results and observed platelet aggregation impairment after levetiracetam administration. Herrman et al., [16] reported a 16 years old girl who developed ecchymosis after starting levetiracetam treatment and persisted until discontinuation of the drug. All hematological parameters were normal including platelets count, clotting factors and von Willebrand factor. There was no history of hematological disorder herself or in her family and she did not use any other medication. The report concluded that the ecchymoses were caused by altered platelet functions.

Aydogan et al., [17] Al-zahrani et al., [6] and Gallerni et al., [18] case reports hypothesized that levetiracetam induced pancytopenia is through myelotoxicity and bone marrow suppression. The myelosuppressive effect of levetiracetam was documented in a case report presented by Peyrl et al., [19] who reported 16 years old boy with acute lymphoblastic leukemia (ALL). The patient developed 
seizures secondary to ALL and was treated with levetiracetam monotherapy. Therefore, he underwent hematopoietic stem cell transplantation from a matched unrelated donor. After one month, the patient developed secondary graft failure. There was no evidence of viral infection or autoimmune manifestations. Levetiracetam was tapered off and replaced by another antiepileptic drug, so the patient showed improvement and the blood cell counts remained stable until discharge.

Other studies suggested the effect of levetiracetam on zinc level which may be the direct cause of levetiracetam-induced anemia based on Aghamollaii et al., [20] and Zou et al., [21] reports. Aghamollaii et al., [20] presented three patients with seizure who developed levetiracetam-induced alopecia which improved after adding zinc sulfate to the therapeutic regimen. Zou et al., [21] reported five patients with epilepsy who developed hair loss with levetiracetam which improved after zinc supplementation.

Folic acid deficiency could be another factor of levetiracetam-induced anemia based on a study on rabbits was done by Praag, [22] who concluded that levetiracetam has induced severe folic acid deficiency. This was conflicted by studies done by Linnebank et al., [23] who conducted their study on 1062 patients on levetiracetam therapy that showed folic acid levels to be within the normal range throughout the study and Gaitatzis et al., [24] study, which was a prospective study on patients treated with different antiepileptic drugs to evaluate the long term adverse events and observed no effect of levetiracetam on folic acid serum levels.

The mode of action of levetiracetam is known to be by binding to synaptic vesicle protein $2 \mathrm{~A}$ (SV2A). Bachman et al., [25] hypothesized thrombocyte expression of synaptic vesicle protein $2 \mathrm{~A}$ (SV2A). Although, thrombocyte expression of synaptic vesicle protein $2 \mathrm{~A}(\mathrm{SV} 2 \mathrm{~A})$ is a matter of debate, it could be a possible mechanism of levetiracetam-induced thrombocytopenia.

Another possibility of levetiracetam induced thrombocytopenia was proposed by Sahaya et al., [26] who speculated that thrombocytopenia could be immune mediated. This hypothesis is supported by Kimland et al., [27] who observed irregular antibodies for platelets in the blood of patient treated with levetiracetam. The bone marrow aspiration showed unremarkable morphology with normal megakaryocytes.

The coagulation profile impairment may be a presentation of liver function impairment as ob- served in Bauer et al., [28] study, who documented in their study the elevation of gamma glutamyl transferase (GGT) in 5.3\% of 505 studied patients secondary to levetiracetam therapy. Another study was done by Sethi et al., [29] reported a case of asymptomatic elevation of liver enzymes due to levetiracetam. The liver enzymes returned back to normal after levetiracetam stoppage. Azar et al., [30] reported a case of elevated liver transaminases after rapid titration of oral levetiracetam which normalized after levetiracetam discontinuation. These effects on liver enzymes are thought to be marker of liver function deterioration in response to levetiracetam therapy and then the coagulation profile of the patients expressed as prolongation of prothrombin and partial thromboplastin time.

Hacquard et al., [31] report suggested that levetiracetam inhibits thromboxane-dependent platelet activation and aggregation. This effect has already been reported for another pyrrolidone derivative molecule, piracetam. Thus, levetiracetam could affect the coagulation profile through thrombocytopenia, prolongation of prothrombin time (PT) and partial thromboplastin time (PTT) or throbasthenia. So, caution should be exercised when levetiracetam is used concomitantly with antiplatelet agents because of an expected additive effect.

There were limitations to this study: The number of studied patients was small, the levetiracetam doses were variable and the lack of control group, which help determination of epilepsy effect itself on hematological parameters. The unavailability of frequent follows-up throughout the study and absence of levetiracetam plasma level monitoring, which help establishing the relation between levetiracetam plasma level and observed adverse effect were other limitations.

In conclusion, our data suggests that levetiracetam monotherapy has proven to be well tolerated in treatment of children with idiopathic epilepsy and does not appear to cause significant changes in complete blood picture, prothrombin time, partial thromboplastin time and platelet functions.

\section{Recommendations:}

Any child treated by levetiracetam should be followed-up with laboratory study periodically; including complete blood picture and bleeding profile. Educating caregivers the warning signs of the expected side effects which require seeking medical advice. More studies with larger sample size and longer duration are needed to find out side effects of levetiracetam in children and search the mechanism of levetiracetam side effects, meth- 
ods of prophylaxis and the effect of levetiracetam withdrawal on side effect elimination. Execution of more studies that study the relation between the side effects, serum level and dose of levetiracetam. Further clinical trials to find out whether the observed alterations are complications from epilepsy itself or side effects from levetiracetam and the tolerability of levetiracetam polytherapy.

\section{References}

1- FISHER R.S., BOAS W.V., BLUME W., et al.: Epileptic seizures and epilepsy: Definitionsproposed by the International League Against Epilepsy (ILAE) and the International Bureau for Epilepsy (IBE). Epilepsia, 46: 4702, 2005.

2- REILLY C., ATKINSON P, DAS K.B., et al.: Neurobehavioral comorbidities in childrenwith active epilepsy: A population-based study. Pediatrics, 133: 1586-92, 2014

3- RAHMAN M.: Use of new generation antiepileptic drugs in children. Bangladesh Journal of Child. Health, 37: 1367, 2013.

4- VIGEVANO F.: Levetiracetam in pediatrics. Journal of Child Neurology, 20:87-93, 2005.

5- HARDEN C.L.: Safety profile of levetiracetam. Epilepsia, 42: 36-49, 2001.

6- ALZAHRANI T., KAY D., ALQAHTANI S., et al.: Levetiracetam-induced pancytopenia. Epilepsy and Behavior, 4: 45-7, 2015

7- OGHLAKIAN R., NOCK C. and KOUBEISSI M.: A case of levetiracetam-induced thrombocytopenia. Epileptic Disorders, 12: 335-7, 2010

8- FRENCH J., EDRICH P. and CRAMER J.A.: A systematic review of the safety profile of levetiracetam: A new antiepileptic drug. Epilepsy Research, 47: 77-90, 2001.

9- GEORGE D. and MALLERY P.: IBM SPSS statistics step by step: A simple guide and reference. 14 th edition, Routledge, USA, 149-55, 2016.

10- DINOPOULOS A., ATTILAKOS A., PASCHALIDOU A., et al.: Short term effect of levetiracetam monotherapy on hematological parameters in children with epilepsy: A prospective study. Epilepsy. Research, 108: 820-3, 2014.

11- TACKE M. and BORGGRAEFE I.: No clinically significant lymphocyte count change in pediatric patients treated with levetiracetam. Epilepsy Research, 114: 147-8, 2015.

12- KANEMURA H., SANO F., TANDO T., et al.: Efficacy and safety of add-on levetiracetam in refractory childhood epilepsy. Brain and Development, 7: 1-6, 2012.

13-GLAUSER T., AYALA R., ELTERMAN R., et al.: Doubleblind placebo-controlled trial of adjunctive levetiracetam in pediatric partial seizures. Neurology, 66: 1654-60, 2006.

14- RÜEGG S., NAEGELIN Y., HARDMEIER M., et al.: Intravenous levetiracetam: treatment experience with the first 50 critically ill patients. Epilepsy and Behavior, 12: 477-80, 2008.

15- MANOHAR C., AVITSIAN R., LOZANO S., et al.: The effect of antiepileptic drugs on coagulation and bleeding in the perioperative period of epilepsy surgery: the Cleveland clinic experience. Journal of Clinical Neuroscience, 18: 1180-4, 2011.

16- HERRMAN H., TAUBØLL E. and GJERSTAD L.: Levetiracetam-induced thrombocytopathia. Epilepsia, 47: 159-60, 2006.

17- AYDOGAN H., YALÇN S., KARAHAN M.A., et al.: Pancytopenia associated with levetiracetam treatment in Lafora's disease. Blood Coagulation and Fibrinolysis, 23: 175-6, 2012

18- GALLERANI M., MARI E., BOARI B., CARLETTI R., MARRA A. and CAVALLO M.: Pancytopenia associated with levetiracetam treatment. Clinical Drug Investigation, 29: 747-51, 2009.

19-PEYRL A., WEICHERT N., KÜHL J., et al.: Levetiracetam as a possible cause of secondary graft failure after allogenic hematopoietic stem cell transplantation. Journal of the European Pediatric Neurology Society, 19: 75-7, 2014.

20- AGHAMOLLAII V., KHAN Z.G., MANESHI A., et al.: Role of zinc supplementation in the treatment of levetiracetam-induced hair loss: A case series. Journal of Pharmaceutical Care, 4: 44-5, 2016.

21- ZOU X., HONG Z. and ZHOU D.: Hair loss with levetiracetam in five patients with epilepsy. Seizure., 23: 158 60, 2014.

22- PRAAG EV.: Drug induced folic acid (vitamin B9) deficiency in a hydrocephalus mini lop rabbit. Medi. Rabbit., 10: 1-10, 2015.

23- LINNEBANK M, MOSKAU S, SEMMLER A, et al.: Antiepileptic drugs interact with folate and vitamin B 12 serum levels. American Neurological Association, 10: $352-9,2011$.

24- GAITATZIS A and SANDER JW.: The long term safety of antiepileptic drugs. CNS Drugs, 27: 435-45, 2013.

25- BACHMANN T., BERTHEUSSEN K.H., SVALHEIM S., et al.: Haematological side effects of antiepileptic drug treatment in patients with epilepsy. Acta Neurologica Scandinavica, 124: 23-7, 2011.

26- SAHAYA K., GOYAL M.K., SARWAL A., et al.: Levetiracetam-induced thrombocytopenia among inpatients: A retrospective study. Epilepsia, 51: 2492-5, 2010.

27- KIMLAND E., OJEBERG B.H., and VON EULER M.: Levetiracetam-induced thrombocytopenia. Epilepsia, 45: 877-8, 2004.

28- BAUER J., BEN-MENACHEM E., KRÄMER G., et al.: Levetiracetam: A long term follow-up study of efficacy and safety. Acta Neurologica Scandinavica, 114: 169-76, 2006.

29- SETHI N.K., SETHI P.K., TORGOVNICK J., et al.: Asymptomatic elevation of liver enzymes due to levetiracetam: A case report. Drug Metabolism and Drug Interactions, 28: 123-4, 2013.

30- AZAR N.J. and AUNE P.: Acute pancreatitis and elevated liver transaminases afterrapid titration of oral levetiracetam. Journal of Clinical Neuroscience, 21: 1053-4, 2014.

31- HACQUARD M., RICHARD S., LACOUR J.C., et al.: H. Levetiracetam-induced plateletdysfunction. Epilepsy Research, 86: 94-6, 2009. 


\section{تأثير العلاج بعقار ليفيتيراسيتام على مكونات الدم الدام العابين

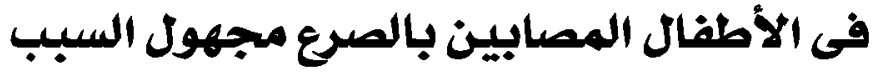

الخخلفية: الصرع هو مرض دماغى يتميز بزيادة تابلية المخ لإنتاج نوبات تشنجية مع وجود مضاعفات عصبية ومعرفية و نفسية وأجتماعية، وهوواحد من أكثر الأضطرابات العصبية لتى تصيب الأطفال شيبوعاً.

الهدف من البحث: تهدف هذه الدراسة لأكتشاف التغيرات الطارئة على صودة الدم الكاملة، زمن البرئرومبين، زمن الثرومبوبلاستين

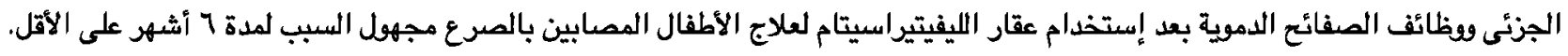

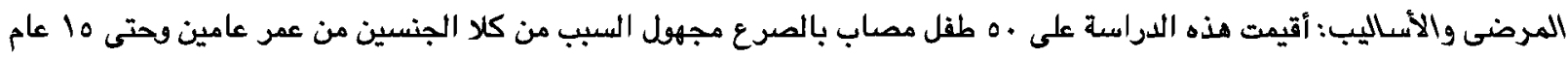

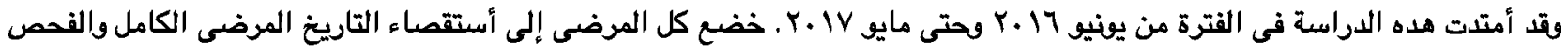

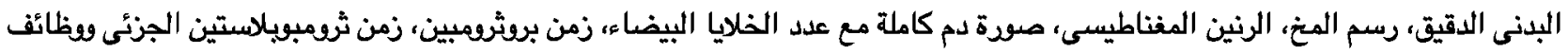

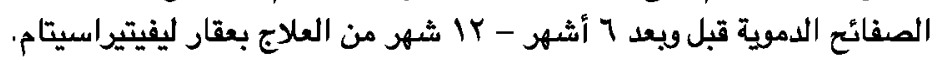

النتائج:وقد أوضحت هذه الدراسة عدم وجود فرق إحصائى فى القيم قيد الدراسة قبل وبعد العلاج. وبناءاً عليه يمكن القول بأن عقار

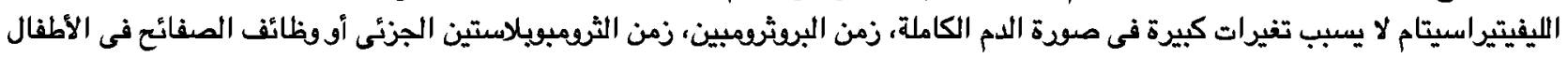

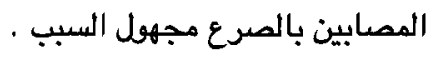

